\title{
我国自由电子激光技术发展战略研究
}

\author{
李鹏，黎明，吴岱，周征，唐淳 \\ (中国工程物理研究院应用电子学研究所, 四川绵阳 621900)
}

\begin{abstract}
摘要: 自由电子激光 (FEL) 光源是一种基于电子直线加速器的大型科研装置, 广泛应用于凝聚态物理、先进材料与表面物理、 原子分子物理、化学、生物等基础科学研究。FEL 技术的进步将促使光源向更高功率和更短波长发展, 以满足日益增长的用 户需求。本文从 FEL 的技术特点出发, 论述了技术发展的必要性, 研判了现阶段国内外 FEL 技术发展现状并凝练我国领域 发展面临的问题。研究提出了我国 FEL 技术的发展思路，在 2035 年前着力在长波波段 FEL、X 射线 FEL、新型 FEL、基于 FEL 的极紫外 (EUV) 光刻光源等方面取得突破。研究建议: 制定科学发展战略、保障科研攻关实施, 加强基础投入、提高 自主创新水平, 加强多方合作、促进科技成果转化, 促进研究与应用结合、推动市场应用, 加强科技人才培养, 以此推进我 国 FEL 领域的稳健发展。
\end{abstract}

关键词：自由电子激光；红外太赫兹光源；极紫外光刻光源；X 射线自由电子激光

中图分类号: TN248.6 文献标识码: A

\section{Development Strategy of Free Electron Laser Technology in China}

\author{
Li Peng, Li Ming, Wu Dai, Zhou Zheng, Tang Chun \\ (Institute of Applied Electronic, China Academy of Engineering Physics, Mianyang 621900, Sichuan, China)
}

\begin{abstract}
Free electron laser (FEL) is a large-scale scientific research device based on electron linear accelerator and has been widely used in basic scientific research such as condensed matter physics, advanced materials and surface physics, atomic and molecular physics, chemistry, and biology. The advancement of FEL technology will promote the light sources to develop towards higher power and shorter wavelength to satisfy the increasing demands of users. This study introduces the necessity of development based on the characteristics of the FEL technology and summarizes the current development status of the technology in China and abroad. It also analyzes the existing problems in its development in China and proposes ideas and goals for the development of FEL technology in China. By 2035, China should focus on making breakthroughs in long-wave FEL, X-ray FEL, new FEL, and FEL-based extreme ultraviolet (EUV) lithography light source technologies. Based on the research and analysis, this study proposes some suggestions for the steady and healthy development of the FEL technology in China, including making scientific development strategies, raising investment in fundamental research to promote independent innovation, strengthening multi-party cooperation to promote the transformation of scientific and technological achievements, promoting market application, and strengthening personnel training.
\end{abstract}

Keywords: free electron laser; infrared-terabertz FEL; extreme ultraviolet-FEL; X-ray FEL

收稿日期 : 2020-03-30; 修回日期 : 2020-05-02

通讯作者: 唐淳, 中国工程物理研究院应用电子学研究所研究员, 研究方向为光学工程; E-mail: tangchun@vip.sina.com 资助项目：中国工程院咨询项目“我国激光技术与应用 2035 发展战略研究” (2018-XZ-27)

本刊网址：www.engineering.org.cn/ch/journal/sscae 


\section{一、前言}

自由电子激光（FEL）是基于真空中自由电子 产生的辐射激光 [1], 与传统激光最主要的区别在 于：辐射波长不依赖于受激介质，仅与电子束的能 量和波荡器 (用于辐射的周期性磁场) 有关。FEL 具有波长完全可调谐的特点, 可以获得其他激光器 不能实现的波段, 相应波长可覆盖从太赫兹至硬 X 射线甚至 $\gamma$ 射线的范围。其中，太赫兹、红外、 极紫外、 $\mathrm{X}$ 射线波长范围内的 FEL 分别称为 $\mathrm{THz}-$ FEL、IR-FEL、EUV-FEL、XFEL。当前, FEL 广泛 应用于凝聚态物理、先进材料与表面物理、原子分 子物理、化学、生物等基础科学研究, 推动了战略 安全、航空航天、能源环境、医药、化工、高端制 造等领域的重大技术革新。

自 1971 年首次提出 FEL 原理 [2] 以来，世界 上建成了 50 多台 FEL 装置, 还有超过 20 台的在建 或拟建装置。科技强国高度重视 FEL 的发展, 投 入重大资源予以保障。尤其是作为新一代探针光的 XFEL $[3,4]$, 使得人类能够首次深入物质内部来实时 观测原子和分子的纳米、微米尺度的演化图像, 进 而操控电子、分子、原子甚至原子核的状态。这类 新型装置促进科学研究方法和模式革新, 有望开辟 新的科学前沿来引发科技和产业革命。例如, 利用 XFEL 诊断核材料及其合金、含能材料在分子 / 原子
层面的时间演化行为，核金属或其合金在高能量密 度状态下的超快动力学行为等。美国建设的国际上 首个 XFEL 装置（LCLS），主要科学目标即为服务 于核物理相关的材料科学研究和高能量密度物理研 究 [5]。相关 FEL 技术也是我国亟待发展的方向 [6]。

本文梳理了 FEL 技术发展的必要性，总结了现 阶段国内外 FEL 技术的发展现状，在凝练我国面临 技术差距的基础上提出了我国 FEL 技术的重点发展 方向, 以期为相关领域的规划发展提供参考。

\section{二、自由电子激光的需求分析}

FEL 装置主要包括加速器、波荡器和光束线系 统三部分（见图 1), 基于加速器产生的相对论电子 束，通过波荡器的周期性磁场产生以受激辐射方式 放大的相干辐射。按照放大增益的不同, FEL 主要 分为低增益和高增益两种机制, 其优点为: (1)波长 任意可调，在理论上不受限制，通过调节电子束能 量和波荡器磁场来改变辐射波长; (2)光束质量好, FEL 辐射在横向是全相干，在纵向是部分相干甚至 全相干, 具有很好的偏振性; (3)峰值和平均功率高, 工作环境为真空, 不存在热处理问题, 理论上不存 在绝对上限。因此, 作为 21 世纪最先进的光源之一, FEL 主要用于产生其他光源不易产生的激光波长、 高峰值功率和高平均功率的激光。

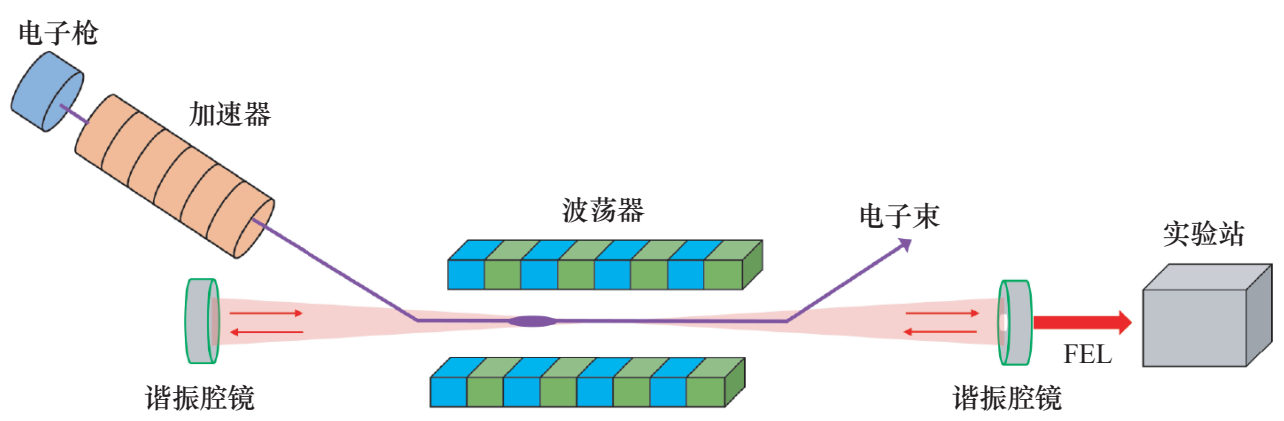

(a) 低增益型FEL

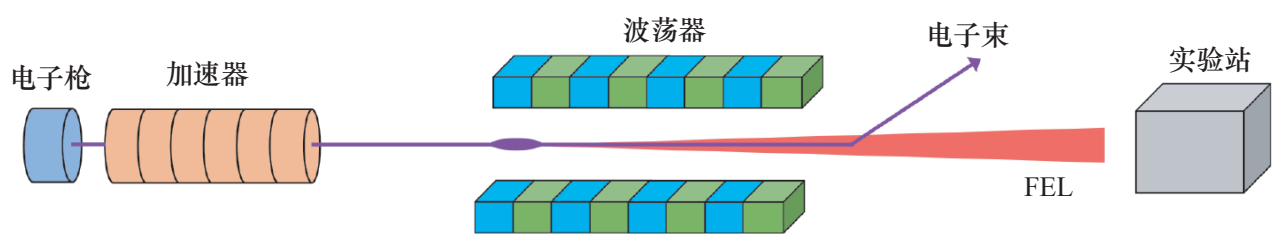

(b) 高增益型FEL

图 1 FEL 装置基本原理 


\section{（一）辐射频率的需求}

在光谱方面, 仍存在着许多传统激光器不易产 生或功率不够的区域，如处于微波与红外之间的太 赫兹波、短波 $X$ 射线甚至 $\gamma$ 射线等，亟需高性能 的光源。FEL 装置是在这些频域内唯一能实现频谱 全覆盖且提供高功率激光的装置, 应用前景广阔, 也是光子科学诸多跨学科应用的关键支撑。

\section{（二）辐射功率的需求}

FEL 装置可提供峰值功率超过 $1 \mathrm{GW}$ 、平均功 率大于 $1 \mathrm{~kW}$ 的高亮度激光, 适应民用工业和国防 安全领域的重大应用需求。极紫外 (EUV) 光刻 技术是芯片制造行业的最前沿技术，需要功率为千 瓦级的新型光源, 而传统的激光等离子体 (LPP) 光源技术发展缓慢。FEL 具有波长可设计、理论 功率极大的特征, 利用 FEL 原理可以产生波长为 $13.5 \mathrm{~nm}$ 的 EUV 光源。国际半导体企业纷纷提出了 采用 FEL 作为 EUV 光源的先进技术方案。

\section{三、国外自由电子激光技术发展现状}

\section{（一）长波波段自由电子激光}

长波波段 FEL 主要延续低增益谐振腔技术路 线, 具有波长可变、功率较高的特点, 被视为激光 武器的一种理想形态, 早期的研究高峰期是 20 世纪 80 年代。由于 FEL 装置系统过于复杂、技术难度 过大, 未能形成相应技术装备。在武器应用背景逐 渐淡出之后，长波波段 FEL 的科研引领作用开始逐 步凸显, 国际上很多装置开始转为利用 FEL 强大功 能开展前沿科学技术及其产业化的研究 (见表 1)。

美国杰斐逊实验室 JLab IR 装置 [7] 起初是作 为激光武器研究发展起来的 FEL 装置, 也是全球 第一台平均功率超过 $10 \mathrm{~kW}$ 的激光器。荷兰 FELIX
装置的建设目标集中在科学研究领域, 是目前全 球覆盖范围最广的用户装置。俄罗斯 NovoFEL 装 置采用低频常温加速器路线导致体积巨大，但成 为目前平均功率最高的运行装置 [8]。德国 FELBE 装置 [9] 是全球长波 FEL 方面性能最完善、用户实 验站数量最多、机时最宝贵、科研产出最高的装 置, 也是第一台基于超导加速器的 THz-FEL 装置。 围绕FELBE 装置开展的应用, 每年发表论文超过 100 篇 (包括《科学》《自然》等期刊论文)。

\section{（二） X 射线自由电子激光}

基于高增益单次放大的 XFEL 是 20 世纪末期 发展起来的新技术 (被称为 “四代光源”), 受到国 际广泛关注。主要发达国家都已建成或在建 XFEL 光源以保障重大科研和国家安全方面的需求（见 表 2)。

美国在 XFEL 领域具有技术优势, 能源部 (DOE) 组织完成了数十次研讨会, 对国家安全、 能源安全、前沿科学领域的需求进行了梳理总结。 以此为指导，美国在 2009 年建成全球首台 XFEL 装置（LCLS）的同时，部署了 LCLS-II、极端条件 下物质与辐射相互作用 (MaRIE) 的建设规划, 形 成全域布局的 XFEL 能力体系。LCLS-II 作为 LCLS 的升级计划, 旨在面向更加宽泛的前沿科学研究需 求 [5]。MaRIE 计划主要面向极端条件下的材料科学 研究, 用于解决核装置、能源安全、前沿科学领域 的国家安全研究需求 (如核装置寿命延长等)。

以德国和俄罗斯为主，包括英国、法国、瑞士 等在内的多国联合开展了 European XFEL 装置建 设 [10]。作为世界上第一台基于超导加速器的高重 频 XFEL 装置, European XFEL 的性能指标引领未 来 10 年发展, 配置了多种极端条件加载装置和与 之配套的诊断技术，成为大国竞相利用的优势科技

表 1 国际上具有代表性的长波波段 FEL 装置

\begin{tabular}{|c|c|c|c|c|c|c|c|c|}
\hline 国家 & 装置 & $\begin{array}{c}\text { 电子能量/ } \\
\mathrm{MeV}\end{array}$ & $\begin{array}{c}\text { 辐射光 } \\
\text { 频率/THz }\end{array}$ & 重复频率 & $\begin{array}{l}\text { 加速器 } \\
\text { 类型 }\end{array}$ & $\begin{array}{l}\text { 典型平均 } \\
\text { 功率/W }\end{array}$ & $\begin{array}{l}\text { 最大峰值 } \\
\text { 功率/kW }\end{array}$ & 出光时间/年 \\
\hline 美国 & JLab IR & $80 \sim 150$ & $21.4 \sim 330$ & 连续多普勒 & $\begin{array}{c}\text { 超导 } \\
\text { (能量回收) }\end{array}$ & 14000 & 250000 & 2005 \\
\hline 荷兰 & FELIX & $15 \sim 45$ & $0.2 \sim 100$ & $10 \mathrm{~Hz}$ & 常温 & 0.4 & 1.5 & 1992、2011 \\
\hline 俄罗斯 & NovoFEL & $10 \sim 44$ & $1.25 \sim 60$ & 连续多普勒 & $\begin{array}{c}\text { 常温 } \\
\text { （能量回收） }\end{array}$ & 100 & 2000 & 2003、2009、2015 \\
\hline 德国 & FELBE & $15 \sim 35$ & $1.2 \sim 75$ & 连续多普勒 & 超导 & 10 & 5000 & 2004、2006、2017 \\
\hline
\end{tabular}


表 2 国际上具有代表性的 XFEL 装置

\begin{tabular}{|c|c|c|c|c|c|c|c|}
\hline 国家 & 装置名称 & $\begin{array}{c}\text { 电子能量 } \\
\text { / } \mathrm{GeV}\end{array}$ & $\begin{array}{c}\text { 辐射光波长 } \\
\text { /nm }\end{array}$ & 重复频率/Hz & $\begin{array}{l}\text { 加速器 } \\
\text { 类型 }\end{array}$ & $\begin{array}{l}\text { 最大峰值 } \\
\text { 功率/GW }\end{array}$ & 出光时间/年 \\
\hline \multirow[t]{2}{*}{ 美国 } & LCLS & 14.3 & $0.08 \sim 1.24$ & 30 & 常温 & 40 & 2009 \\
\hline & LCLSII & 4.0 & $0.25 \sim 6.20$ & 120 & 超导 & - & 未出光 \\
\hline 德国 & European XFEL & 17.5 & $0.04 \sim 0.15$ & 10 & 超导 & 110 & 2017 \\
\hline 瑞士 & SwissFEL & 5.8 & $0.10 \sim 7.00$ & 100 & 常温 & 21 & 2017 \\
\hline 日本 & SACLA & 8.0 & $0.06 \sim 2.82$ & 60 & 常温 & 106 & 2011 \\
\hline 韩国 & PAL-XFEL & 10.0 & $0.10 \sim 10.00$ & 60 & 常温 & 42 & 2016 \\
\hline
\end{tabular}

资源。美国的核技术实验室也在联合申请成为高能 密度实验站 (HED) 的用户。

此外, 瑞士根据本国在生物、医药、纳米材 料等领域的研究需求, 独立建设了 SwissFEL 装置 [11]; 日本、韩国也分别建设了 XFEL 装置 $[12,13]$ 。

\section{（三）新型自由电子激光}

除了传统的基于静磁波荡器的XFEL 装置以外, 基于光学波荡器的新型小型化 XFEL 光源技术成为 研究热点。相关思路早在 20 世纪七八十年代便已 提出, 但受限于当时的技术水平而难以实现。近年 来, 激光技术的蓬勃发展为实现低成本、紧凑型 的 XFEL 提供了新的机遇。基于光学波荡器的量子 FEL 装置, 在输出光子能量、光源相干度、带宽等 特性上突破了传统 XFEL 装置的性能边界, 将成为 XFEL 有益且有力的技术补充。

\section{四、我国自由电子激光技术的发展现状}

\section{（一）取得的成就}

我国 FEL 技术的发展也经历了从国防需求牵引 向科学研究应用的转换过程。我国在 FEL 领域的理 论与实验研究始于 20 世纪 80 年代, 早期代表性的 工作有: 拉曼型 FEL 出光 (1985 年), 波荡器自发 辐射 (1986 年), 谐振腔型技术路线和放大器型技 术路线的 FEL 出光 (1993 年)。2000 年以来, 受到 光子科学对光源装置的需求牵引, 国内新增建设了 一批 FEL 光源装置 (见表 3 )。

在低增益谐振腔型长波 FEL 光源方面, 中国 科学技术大学国家同步辐射实验室 (NSRL) 主要 开展振荡器 FEL 的相关理论和实验研究, 现阶段 重点进行 “基于可调谐红外激光的能源化学研究 大型实验装置” 国家重大科研仪器研制项目的研
究, 后续将建设覆盖中红外到远红外波长范围的可 调谐红外光源（FELiChEM）[14]。华中科技大学重 点开展振荡器 THz-FEL 装置研究。中国工程物理 研究院开展了系列化的振荡器 FEL 理论与实验研 究, 2005 年实现了能量为 $30 \mathrm{MeV}$ 、远红外/太赫 兹 FRL（FIR-FEL）的实验出光 [15]; 2017 年建成 了我国唯一的基于超导加速器的高平均功率 $\mathrm{THz}-$ FEL 装置（CTFEL）[16]。

在高增益短波长 FEL 光源方面, 我国 2000 年 前后开始发展, 已建成的装置覆盖了从紫外到软 X 射线波段。2009 年, 中国科学院上海应用物理研究 所建成了我国首个高增益 FEL 综合研究平台 上海深紫外自由电子激光装置 (SDUV-FEL) [17]。 2016 年, 中国科学院大连化学物理研究所建成 的 “基于可调极紫外相干光源的综合实验研究装 置” 实现出光 [18]。上海应用物理研究所和北京大 学承担的软 X 射线 FEL 项目于 2014 年启动, 建设 波长为 $8.8 \mathrm{~nm}$ 、光子能量为 $0.14 \mathrm{keV}$ 的 FEL 装置, 2020 年 1 月实现了全相干 FEL 输出 [19]。

在 FEL 装置的建设规划方面, 中国工程物理研 究院正在开展长波长的西部光源项目论证; 大连化 学物理研究所拟建设基于超导加速器的连续 EUVFEL 装置。上海科技大学和中国科学院上海高等研 究院正在建造我国首台硬 X 射线 FEL 装置, 配置 了能量为 $8 \mathrm{GeV}$ 的高品质电子束连续波超导直线加 速器, 辐射波长能量为 $0.4 \sim 25 \mathrm{keV}, \mathrm{X}$ 光脉冲最高 重复频率可达 $1 \mathrm{MHz}$, 对促进我国光子科学的发展 具有重要的意义 [20]。

\section{（二）面临的问题}

1. 战略目标待确立

发展 FEL 装置的目的在于建设传统激光器所不 易产生或功率不够的新型光源装置; 而作为大型科 
表 3 我国主要的 FEL 装置

\begin{tabular}{|c|c|c|c|c|}
\hline 装置名称 & 地区 & 辐射光波长 & 加速器类型 & 状态 \\
\hline 北京自由电子激光装置（BFEL） & 北京 & $5 \sim 50 \mu \mathrm{m}$ & 常温 & 退役 \\
\hline 基于可调谐红外激光的能源化学研究大型实验装置 (FELiChEM) & 合肥 & $2.5 \sim 200 \mu \mathrm{m}$ & 常温 & 在建 \\
\hline 高平均功率太赫兹自由电子激光装置（CTFEL） & 成都 & $71.4 \sim 428.5 \mu \mathrm{m}$ & 超导 & 运行 \\
\hline 西部光源红外太赫兹自由电子激光装置（WILL） & 成都 & $2.4 \sim 3000 \mu \mathrm{m}$ & 超导 & 规划 \\
\hline 大连极紫外相干光源装置（DCLS） & 大连 & $50 \sim 150 \mathrm{~nm}$ & 常温 & 运行 \\
\hline 高重复频率软件 X 射线自由电子激光装置 & 大连 & 紫外 软 X 射线 & 超导 & 预研 \\
\hline 上海深紫外自由电子激光装置（SDUV-FEL） & 上海 & $150 \sim 350 \mathrm{~nm}$ & 常温 & 运行 \\
\hline 软 X 射线自由电子激光试验装置（SXFEL） & 上海 & $2 \sim 20 \mathrm{~nm}$ & 常温 & 运行 \\
\hline 硬 X 射线自由电子激光装置（SHINE） & 上海 & $0.05 \sim 3.1 \mathrm{~nm}$ & 超导 & 在建 \\
\hline
\end{tabular}

研装置, FEL 装置将是一个城市甚至一个地区的科 研创新名片。我国在相关领域的宏观战略规划不够 清晰, 不利于发挥大型装置的集群效应和示范效应, 对相应地区的科研布局和创新发展牵引不够。

\section{2. 关键部件技术待突破}

FEL 的诸多理论技术需要探索, 与工程应用密 切相关的关键部件国产化研制仍有待突破, 包括: 加速器与波荡器物理设计与关键问题, 高重频高亮 度光阴极电子枪，超导加速、射频低电平控制、飞 秒同步、高重频束测、超导波荡器、光束线等技术, 用户实验站系统等。

\section{3. 产业化应用待拓展}

现阶段的 FEL 应用集中在科研探索方面，相 关装置的投资建设主要是国家大型科研设备资助 渠道。工业应用类别有限、“产学研”有所割裂的 现状，制约了未来 FEL 技术与应用的进一步发展， 这就使得拓展 FEL 的产业化应用成为领域发展函需。

\section{五、我国自由电子激光技术发展思路}

\section{(一) 发展目标}

以满足国民经济和国防建设的自主可控战略需 求为根本出发点, 围绕 FEL 在前沿基础科学、能源 安全和国防安全等领域的技术需求, 提出关键技术 自主突破和核心部件国产化的发展目标。通过政策 引导、强化创新、应用融合等手段, 建设更高功率、 更短波长的 FEL 光源, 在保障重大科研需求的基础 上拓展实施规模化的工业应用。参与全球科技竞争 并建立相对技术优势, 推动以 FEL 光刻应用为代表 的高端工业应用。

\section{（二）发展路径}

\section{1. 长波波段自由电子激光技术}

研究提出 [21]: 生物神经信号的物理场应为太 赫兹到红外的高频电磁场, 最可能频率范围应在太 赫兹到百太赫兹; 可以把 $0.5 \sim 100 \mathrm{THz}$ 的电磁波称 为太赫兹电磁波, 把有关研究可称为太赫兹生物学。 除了生物学之外, 其他领域对相关频段的光源需求 也很迫切, 但在该频段缺乏高平均功率且频率可调 的光源。

构建覆盖整个红外太赫兹频段的高平均功率可 调谐 FEL 光源, 对于前沿科学研究具有重大意义。 以红外太赫兹 FEL 科学装置为依托, 构建集成强太 赫兹光辐照、强磁场、低温、高压等极端外场和实 验条件、与先进测量技术相结合的综合性平台。相 关平台用于支撑凝聚态物理、新材料、先进光学、 光电子学、生物、医学、水科学等前沿热点研究, 以国家科研需求为导向, 在应用领域拓展后支持开 展多学科、多领域的工业应用。

\section{X 射线自由电子激光技术}

根据国际 XFEL 装置的发展趋势, 提出比国际 上已建和待建 XFEL 装置更加先进的发展目标（与 美国 MaRIE 规划相当), 适时开展我国高重频、高 光子能量 XFEL 装置建设。匹配国家战略安全、航 空航天、能源技术等领域涉及的极端条件材料性能 研究需求, 规划新一代 XFEL 装置, 科学配置实验 线站, 提供与多领域研究需求相配套的诊断技术。

\section{3. 新型自由电子激光技术}

当前, 国际上量子 FEL 技术仍处于原理验证 和探索研究阶段 [22], 但在装置规模、建设成本等 方面具有潜在优势, 可以采用这一技术路线来构建 
实验室规模的小型硬 $\mathrm{X}$ 射线相干光源，用于支持科 研院所和高等院校的科学研究需求。我国适时开 展量子 FEL 技术的发展规划, 注重理论研究并配 套验证实验，在与国际量子 FEL 研究 “同台竞技” 的同时，逐步满足国内日益增长的高性能 X 射线 源的应用需求。

\section{4. 基于 FEL 的 EUV 光刻光源技术}

FEL 的工业应用是其主要发展方向之一，特 别是作为 EUV 光刻光源 [23]。荷兰阿斯麦尔公司 (ASML)、日本 EUV-FEL 光源产业化研究会、美国 格罗方德半导体股份有限公司（Global Foundries） 等企业积极关注 EUV-FEL 光刻技术，认为将是光 刻在 $3 \mathrm{~nm}$ 节点之后的重要技术路线。

在新时期对关系国计民生的关键技术全面 “自 主可控” 的强烈需求下, EUV 光刻技术将是我国 亟待解决的 “卡脖子” 技术之一，也是我国未来芯 片发展的重要基础。按照关键技术验证、原理样机 研制、产业化布局的 “三步走” 路线，规划我国 EUV-FEL 发展路线, 推动 EUV-FEL 产业化发展。

\section{六、对策建议}

\section{（一）科学制定发展战略，保障科研攻关实施}

我国 FEL 技术研究处于蓬勃发展时期，相关 机构提出了多个 FEL 装置的建设意向。建议提前论 证和制定较为详尽的发展策略与研发目标，注重需 求牵引、突出应用导向，通过科学的规划布局来推 动 FEL 领域的良性循环和可持续发展。FEL 装置 属于大型科研装置, 应注重强强联合、加强组织管 理，保证建设项目按质按期完成并发挥预期的关键 作用。

\section{（二）加强基础投入，提高自主创新水平}

FEL 技术属于科技基础研究，装置系统复杂、 投资大、周期长。建议政府保障 FEL 科研资源投入, 通过项目建设和装置运行的牵引，引导相关行业和 企业、科研院所、高等院校的探索创新与应用研究 方向。同时，通过各类基金的定向资助，推动关键 部件的全面国产化; 鼓励领域科研创新和新原理新 技术的出现，突破 FEL 装置小型化和轻量化技术， 促进 FEL 技术及其应用的健康发展。
（三）加强多方合作, 促进科技成果转化

FEL 装置涉及多个领域, 国际上 FEL 建设较 多采用多方合作的形式：项目实施由牵头机构总负 责、多家科研机构共同参与，相关设备由科研机构 研发、企业协作制造。这种方式有利于整合科技研 发力量协同开展技术攻关，同时充分发挥优势企业 的加工制造能力, 在装置建设过程中实现技术转化。 建议在我国 FEL 领域加强行业间的技术合作，切实 推动科研机构与优势企业的分工合作, 通过 “产学 研”联合来促进 FEL 领域的综合发展。

\section{（四）促进研究与应用结合，推动市场应用}

工业应用是 FEL 技术发展的重要方向，建议将 以 EUV 光刻光源为引领来实现 FEL 技术的产业化 转化作为示范项目。通过项目实施, 消除 “产学研” 脱节现象，探索形成极具价值的产业化方案，为后 续技术转化应用积累经验、指明方向。加快 EUV 光刻光源技术方案与产业发展的论证, 鼓励申请优 质专利并加强知识产权管理。引入企业力量建立样 机研制平台，推动 FEL 产业的市场示范应用与规模 化发展。

\section{（五）营造文化氛围，加强人才队伍培养}

支持和鼓励科研机构参与国际科技合作计划、 国际大科学计划和大科学工程, 承担或组织国际重 大科技合作项目。引导有条件的高等院校和科研院 所建立专业化、市场化的技术转移机构，注重科技 成果发布。培养一批高层次领军人才和骨干专业技 术人才，加快推进 FEL 相关学科的人才发展和机制 创新。加大高端人才引进力度, 开展国际性的 FEL 技术交流，全面提升人才队伍建设与国际科技合作 的质量。

\section{参考文献}

[1] 金光齐, 黄志戎, 瑞安·林德伯格. 同步辐射与自由电子激 光一一相干X射线产生原理 [M]. 北京: 北京大学出版社, 2018. Kim K J, Huang Z R, Lindberg R. Synchrotron radiation and free-electron lasers: Principles of coherent X-ray generation $[\mathrm{M}]$. Beijing: Peking University Press, 2018.

[2] Madey J M. Stimulated emission of bremsstrahlung in a periodic magnetic field [J]. Journal of Applied Physics, 1971, 42: 1906 1971.

[3] Green B, Kovalev S, Asgekar V, et al. High-field high-repetition- 
rate sources for the coherent $\mathrm{THz}$ control of matter $[\mathrm{J}]$. Scientific Reports, 2016, 6: 1-9.

[4] Feng C, Deng H X. Review of fully coherent free-electron lasers [J]. Nuclear Science and Techniques, 2018, 29(11): 1-23.

[5] Yabashi M, Tanaka H. The next ten years of X-ray science [J] Nature Photon, 2017, 11(1): 12-14.

[6] 赵振堂, 冯超. X射线自由电子激光 [J]. 物理, 2018, 47(8): 481490.

Zhao Z T, Feng C. X-ray free electron lasers [J]. Physics, 2018, 47(8): 481-490.

[7] Carr G L, Martin M C, McKinney W R, et al. High-power terahertz radiation from relativistic electrons [J]. Nature, 2002, 420(6912): 153-156.

[8] Vinokurov N, Arbuzov V S, Chernov K N , et al. Novosibirsk high-power THz FEL facility [C]. Saint Petersburg: 2016 International Conference Laser Optics, 2016.

[9] Bostedt C, Boutet S, Fritz D M, et al. Linac coherent light source: The first five years [J]. Reviews of Modern Physics, 2016, 88(1): $1-10$.

[10] Weise H, Decking W. Commissioning and first lasing of the European XFEL [C]. Santa Fe: 38th International Free Electron Laser Conference, 2017.

[11] Milne C, Schietinger T, Aiba M, et al. SwissFEL: The Swiss X-ray free electron laser [J]. Applied Sciences, 2017, 7(7): 720.

[12] Ishikawa T, Aoyagi H, Asaka T, et al. A compact X-ray freeelectron laser emitting in the sub-ngstrm region $[\mathrm{J}]$. Nature Photonics, 2012, 6(8):540-544.

[13] Kang H S, Min C K, Heo H, et al. Hard X-ray free-electron laser with femtosecond-scale timing jitter [J]. Nature Photonics, 2017, 11(11): 708-714.

[14] Zhao Z Y, Li H T, Jia Q K. Effect of cavity length detuning on the output characteristics for the middle infrared FEL oscillator of
FELiChEM [J]. Chinese Physics C, 2017, 41(10): 1-6.

[15] Jin X, Li M, Xu Z. Experiment study on the CAEP FIR-FEL [J]. Chinese Physics C, 2006, 30(1): 96-98.

[16] 黎明, 杨兴繁, 许州, 等. 太赫兹自由电子激光的受饱和实验 [J]. 物理学报, 2018, 67(8): 1-9.

Li M, Yang X F , Xu Z, et al. Experimental study on the stimulated saturation of terahertz free electron laser [J]. Acta Physica Sinica, 2018, 67(8): 1-9.

[17] Zhao Z T, Wang D, Chen J H, et al. First lasing of an echo-enabled harmonic generation free-electron laser $[\mathrm{J}]$. Nature Photonics, 2012, 6(6): 360-363

[18] Wang H L, Yu Y, Chang Y, et al. Photodissociation dynamics of $\mathrm{H}_{2} \mathrm{O}$ at $111.5 \mathrm{~nm}$ by a vacuum ultraviolet free electron laser [J]. The Journal of Chemical Physics, 2018, 148(12): 1-15.

[19] Zhao Z T, Wang D, Gu Q, et al. Status of the SXFEL project [J]. AAPPS Bulletin, 2016, 26(1): 12-24.

[20] Zhu Z Y, Zhao Z, Wang D, et al. SCLF: An 8-GeV CW SCRF linac-based X-ray FEL facility in Shanghai [C]. Santa Fe: Proceedings of the 38th International Free-Electron Laser Conference, 2017.

[21] 刘国治. 关于生物神经系统物理机理的若干猜想 [J]. 科学通报, 2018, 63(36): 16-17.

Liu G Z. The conjectures on physical mechanism of vertebrate nervous system [J]. Chinese Science Bulletin, 2018, 63(36): 1617.

[22] Bonifacio R, Fares H, Ferrario M, et al. Design of a sub-angstrom compact free-electron laser source [J]. Optics Communications, 2017, 382(1): 58-63.

[23] Kawata H. Challenges to realize the EUV-FEL high power light source - Present status on the EUV-FEL R\&D activities [C] Berkeley: 2017 International Workshop on EUV Lithography, 2017 